\title{
FIELD EXPERIMENTATION OF COTS-BASED UAV NETWORKING
}

\author{
Dan Hague \\ Air Force Research Laboratory \\ Rome, New York, USA \\ daniel.hague@rl.af.mil
}

\author{
H. T. Kung \\ Harvard University \\ Cambridge, MA, USA \\ kung@harvard.edu
}

\author{
Bruce Suter \\ Air Force Research Laboratory \\ Rome, New York, USA \\ bruce.suter@rl.af.mil
}

\begin{abstract}
Low-cost and high-performance Commercial Off-TheShelf (COTS) wireless equipment, such as IEEE 802.11 wireless LAN ("Wi-Fi"), has so advanced that it is now practical to use it in small low-altitude Unmanned Aerial Vehicles (UAVs). This new capability has inspired many novel application ideas in UAV networking. We argue that field experimentation of $U A V$ networking is essential in collecting link measurement data, developing network protocols and applications, and evaluating their performance in realistic environments, and that it is feasible to conduct these experiments cost-effectively with COTS-based equipment. We describe several ongoing field experiments and initial results. Lastly, we briefly describe future testing plans as well as suggest methods of facilitating rapid and inexpensive UAV networking field experimentation.
\end{abstract}

\section{INTRODUCTION}

Potential applications of COTS-based UAV networking for low-cost small UAVs are abundant. For example, UAVs could act as relays between ground objects that could not otherwise communicate due to distance or lack of line-of-the-sight; multiple UAVs could simultaneously record and track the count of wildfires; and UAV networking could create an instant communication infrastructure following a disaster or during sporting events. But uncertainties with these networks are also abundant. Beyond usual quality of service concerns about wireless mobile networks, there are UAV and COTS specific issues such as dynamically changing link quality due to UAV's movement and banking, and relatively low tolerance [5] of low-cost COTS wireless receivers to radio interferences.

At present, the best practices of COTS-based UAV networking and their expected performance in various airborne applications are not well-understood. Literature in this area is scarce. This is partly due to the relative newness of low-cost COTS-based radio and networking apparatuses (e.g., 802.11 equipment) and partly due to the fact that past work on small UAVs mainly focused in other areas such as UAV control (e.g., [1]) and single-plane UAV applications (e.g., [8]). As a result, it is unclear, for example, how well 802.11 COTS equipment, which was originally designed to provide local wireless access to the Internet for laptops and desktops, would work in UAV networking

In this paper we argue that field experiments are essential in improving our knowledge in COTS-based UAV networking. This is because real-world UAV networking and the environments in which it expects to operate are far too complex to be addressed by other means (e.g., simulation or modeling alone). For instance, it would be necessary to carry out field experiments in collecting link measurement data, developing network protocols and applications, discovering networking system issues (e.g., tradeoff between throughput and latency) and validating solutions. Furthermore, we argue that by using COTS communications equipment and UAV platforms, relatively quick and inexpensive field experiments are feasible. We suggest methods that can facilitate such rapid and low-cost field experimentation of UAV networking. For illustration, we describe some ongoing field experiments that we are conducting.

\section{DESIGNING FIELD EXPERIMENTS FOR UAV NETWORKING}

There are a number of issues regarding the design of field experiments for UAV networking. As discussed below, these include considerations concerning link measurement, communications and networking protocols, test payload, and applications.

\section{Design of Link Measurement Experiments}

In developing UAV networking applications and protocols, it is important that we can characterize UAV wireless links under various environments of interest. For example, we need to know link performance 
against metrics such as Receive Signal Strength Indication (RSSI), UDP throughput and packet loss rates.

The communications environment where a UAV network operates is usually much too complex to be captured by simple models, such as free-space propagation models. Wireless links of a UAV may exhibit varying quality over time due to a variety of factors, including changes in communication distance, antenna polarization caused by airplane's banking, direction of the communicating party in the antenna radiation pattern, shadowing resulting from blocking of line-of-sight by on-board electronic equipment, ground reflection and Doppler effects. Furthermore, when a UAV communicates with nodes on the ground, multi-paths caused by reflections from ground as well as nearby objects, such as trees, hills, buildings and vehicles, can affect the quality of the communication channel. These objects may sometimes block line-of-sight. In addition, there could be radio interferences from other ground transmitters in the region operating at the same or adjacent frequency bands. This interference problem can be especially serious for 802.11 equipment, given its widespread deployment.

For these reasons, it is necessary to conduct UAV field experiments to collect measurement data that can accurately characterize a UAV's wireless communication links in environments of interest. The design of such experiments will involve the design of the UAV fly (e.g., altitude, speed and flight pattern), position and elevation of ground nodes, type and orientation of antennas on the UAV and ground nodes, traffic load (e.g., selection of traffic source and destination nodes, uni- or bi-directional transmission, transmit rate and packet size), etc.

\section{Design of Protocol Experiments}

Consider, for example, a multi-hop UAV network, where packets are relayed by one or more UAVs. Such a multi-hop relaying network can extend network range and provide communication beyond line of sight.

There is a large design space for multihop protocols, ranging from the traditional single-radio, singlechannel protocol based on CSMA/AD to multi-radio multi-channel protocols based on CSMA/CD, TDM or FDM [5]. At the physical layer, we need to ensure sufficiently high Signal to Interference plus Noise
Ratio (SINR) for each hop, in the presence of possible radio interference from the neighboring hops [9] and adjacent channel interference (ACI) from the same node [4][10]. At the MAC layer, in selecting a proper wireless link to use a node may send probe packets to test link quality. At the network layer, unlike conventional hierarchical routing for the wired Internet, routing for ad-hoc UAV networks likely needs to be flat. That is, all nodes are on the same level and perform routing functions. For example, every node must maintain or discover routes to the destination [7].

In a UAV network which experiences rapid change of link conditions, the chance that all links on a multihop path are in good conditions at the same time is likely to be small. Future experiments will characterize the probability and characteristics of this multihop environment. In this case, it would be useful for a relay UAV to buffer outgoing packets when its outgoing link experiences poor link conditions. This buffering method would be similar to the Delay Tolerant Networks (DTN) approach [3].

For these reasons, we need to design UAV networking experiments to explore protocol design space, and evaluate new approach such as DTN-like protocols.

\section{Design of UAV Test Payload}

While these aforementioned issues concerning communication links and networking protocols could be complex, solutions must nevertheless be simple, lightweight and flexible. Specifically, they need to satisfy physical constraints imposed by a small UAV, with respect to form factor, weight, battery budget, etc.

For example, a small UAV based on a 96" wingspan Senior Telemaster (see Figure 2 and 3) can afford only a networking payload of low single digit pounds, while carrying fuel or batteries sufficient for a half to one hour flight. In addition, there is space constraint that only allows a processor board of size not larger than 5"x8". Within the space constraint, accessories such as bulk data storage, radio cards, and batteries also need to be accommodated. Some applications would require the inclusion of other payload components such as GPS and camera.

Beyond satisfying physical constraints of a small $\mathrm{UAV}$, the payload design typically needs to meet some additional objectives. For example, the design 
may need to be flexible to allow frequent component changes, friendly in payload access to facilitate field operation, and ruggedized to endure aircraft's shaking and temperature variations.

An integrated part of the networking payload is the placement of radio antennas and their cables. These antennas are for radio control (R/C) of the airplane and for its data communications (e.g., 802.11 radios). The R/C antennas would need to be placed sufficiently far from the on-board processor board to reduce interferences. In one of our early payload designs, we observed serious interference on a $72 \mathrm{MHz}$ $\mathrm{R} / \mathrm{C}$ receiver by a $100 \mathrm{MHz}$ processor board on the UAV. We later used a multi-prong approach to mitigate the interference problem: (1) moving the $\mathrm{R} / \mathrm{C}$ equipment including receiver and antenna, to the tail of the airplane, (2) shielding the box hosting the processor board with metal screen wrap, and (3) replacing an external power switch of the processor board with an internal switch to minimize radio frequency interference. For data communications we have been using custom-made dipole antennas that can be conveniently mounted at desired locations of the UAV wings.

\section{Design of Application Experiments}

Through application-level experiments, we can evaluate the overall end-to-end application performance of a UAV networking system. Application experiments could be, for example, a UAV's retrieving of sensing data from sensor nodes on the ground, a UAV's relaying of packets for ground networks, and UAV-toUAV multi-hop packet or message relaying. It is useful to focus on essential mechanisms shared by multiple applications. For example, for UAV packet relaying and data retrieving applications, we could design experiments to evaluate relay and retrieving mechanisms, respectively.

A key to the success of these application mechanisms is their ability of adapting to a UAV's dynamically varying link quality. For example, it would be desirable that the encoding of video taken by a UAV can dynamically adapt to the condition of the UAV's output link. That is, the encoder will produce a high-rate video stream when the link is good and a low-rate one otherwise.

\section{FIELD EXPERIMENT CASES}

We describe two of our ongoing COTS-based field experiments in UAV networking. The first experiment uses 802.11 equipment, whereas the second one uses $900 \mathrm{MHz}$ technology. The two experiments complement each other in the sense the former and the latter address high-bandwidth and long-range communications and networking needs, respectively. Future experiments will integrate both systems and use them simultaneously.

\section{Case 1: UAV Networking with COTS 2.4GHz and 5GHz 802.11}

We have conducted field experiments for UAV networks based on $2.4 \mathrm{GHz}$ and $5.8 \mathrm{GHz}$ COTS 802.11 equipment for the past nine months. The experimentation has progressed through a number of stages. Experiments were first performed in labs, then in some large indoor spaces (e.g., a gym) and open outdoor grounds, and finally, in an airfield. Network nodes were first placed on the ground, then on antenna towers and balloons, and finally, on UAVs. Through these stages of experiments, we calibrated our network equipment, refined system software, improved the design of our UAV networking payload, wrote various scripts for running experiments, and developed data analysis and visualization tools.

Figure 1 is a flight path plot of a UAV run in a set of experiments conducted in April 2006. The purpose of the experiment was to measure link quality for communication between a UAV and ground nodes, under various communicating distances and antenna configurations. The UAV used was a gas-powered model airplane (Senior Telemaster). In the diagram persecond UAV positions, obtained by a GPS receiver mounted on the UAV, are shown as dots on the UAV flight path. The UAV flew approximately at 50-yard altitude and at 40 miles per hour speed over four ground network nodes. Three of the ground nodes had their antennas placed about 12" above the ground, whereas the fourth one was mounted on the top of a 14 -ft wooden pole. 


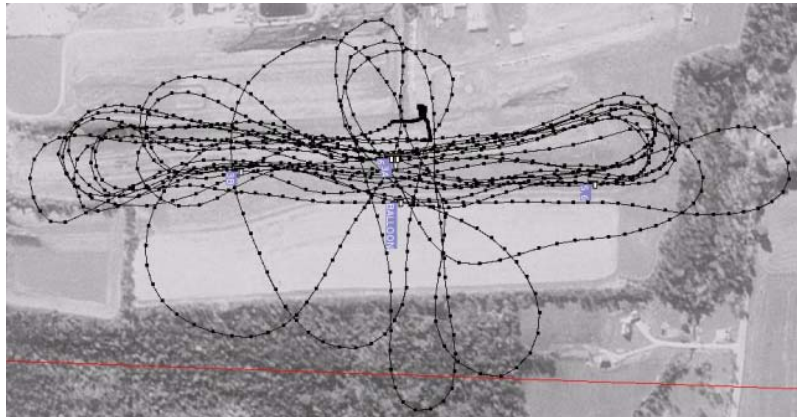

Figure 1. UAV flight path recorded by an on-board GPS receiver

In one of these experiments, the UAV was equipped with two radios each with two antennas. These four antennas were mounted on the UAV wings (see Figure 2) in the three orientations: (1) "vertical to the ground", (2) "horizontal to the ground and parallel to the flight direction" and (3) "horizontal to the ground and perpendicular to the flight direction". Using these antennas one at a time in a round-robin manner, the UAV broadcast data packets to the four ground nodes. Each ground node was equipped with two radios each with its own antenna. Both radios of a ground node simultaneously receive packets broadcast by the UAV. Our measurements on throughput suggest that the pair of horizontal antennas that are orthogonal to the flight path perform better than others for most of UAV positions (see [6] for a detailed accounting of the measurement results). Based on these results, we can devise strategies for dynamic and automatic selection of optimal antenna pair to use during a UAV flight.

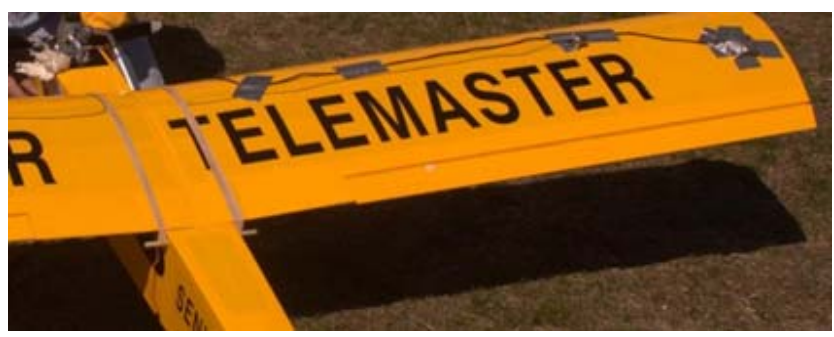

Figure 2. Custom-made dipole antennas mounted on a UAV wing

In a recent UAV flight test, with our custom-made antenna, we were able to demonstrate greater than one mega-bit-per-second throughput in a three-hop network involving two relay UAVs.
In another experiment, we compared range reachable by $802.11 \mathrm{a}$ with that by $802.11 \mathrm{~g}$. Generally speaking, with $802.11 \mathrm{~g}$ the UAV can communicate to ground nodes easily even when it is as high as $600 \mathrm{ft}$ above the ground. Thus with $802.11 \mathrm{~g}$ robust communication between UAVs and ground nodes is feasible. In [2] similar results were reported for $802.11 \mathrm{~b}$. These results have led to some of our current application experiments focusing on evaluating networking performance between UAV and ground nodes. To enhance the robustness of the communication, we are investigating the use of ground node clusters as relays. That is, any node in a cluster can receive broadcast packets from a UAV and can transmit packets for the next hop. These clusters can thus increase redundancy in the relay operation so as to improve the relay reliability and throughput.

\section{Case 2: UAV Networking with COTS $900 \mathrm{MHz}$ Technology}

Preliminary runs of a second set of experiments has been accomplished using COTS $900 \mathrm{MHz}$ technology in a point to point mode. The focus of this set of experiments is the development of mechanisms and tools for the collection of data characterizing the performance of various communications link technologies. As with the previously discussed 802.11 experiments, a Senior Telemaster (see Figure 3) was used and these experiments were initially developed and prototyped in a laboratory environment.

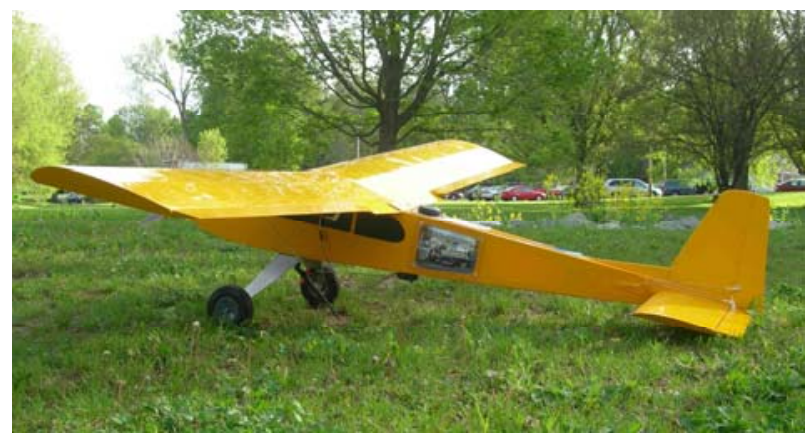

Figure 3. Electric powered Senior Telemaster with a $900 \mathrm{MHz}$ serial RF modem hosted in the middle section of the fuselage

\section{MHz Serial Modem}

As depicted by Figure 4, the payload for this set of experiments consists of a small Soekris processor 
board running Linux, a COTS 900MHz serial RF modem (Microhard Systems' MHX-920), and a small GPS receiver. The processor sends test data including GPS information to a corresponding processor at a ground location. The ground node calculates and logs effective throughput, packets in error, total packets, RSSI and GPS information. This information is displayed on a ground workstation and logged for later processing. GPS information is also passed to an external application for real time display of platform position tracking.

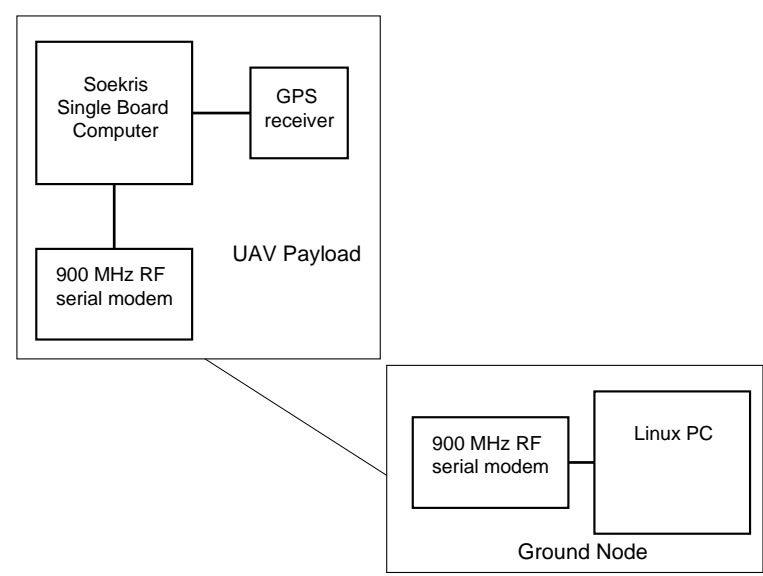

Figure 4. $900 \mathrm{MHz}$ serial modem test onfiguration

Minimal testing has been done to date in determining how RSSI and throughput vary as a function of communication distance. For these tests the UAV's distance from the ground receive antenna is roughly 8 miles. Altitude of the platform ranged from approximately 350 - 900 feet. More testing is needed to gather enough data to draw relevant conclusions, but the use of small COTS platforms and equipment has supported the ability to easily collect the necessary data.

\section{MHz 802.11}

We have also tested another $900 \mathrm{MHz}$ approach using the recent Atheros' $900 \mathrm{MHz}$ wireless solution based on the 802.11 protocols. Our UAV field experimentation has found that this solution has significantly longer communication ranges than $802.11 \mathrm{a} / \mathrm{b} / \mathrm{g}$, while still being able to achieve megabits per second bandwidth. The long range property of this technology under a 1-megabit-per-second transmitter is clearly demonstrated in Figure 5. Ranges of up to 2000 meters were attained from aircraft to ground vehicle, in a tree filled rural environment.

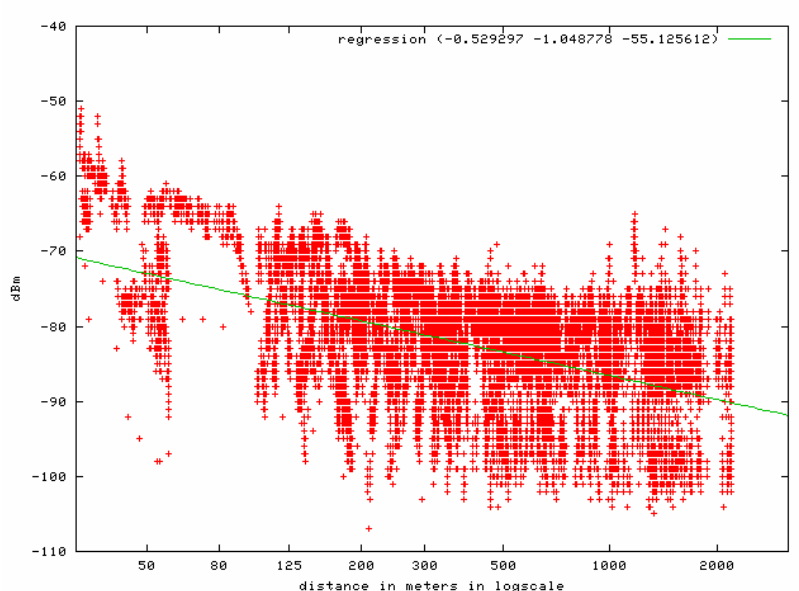

Figure 5. RSSI (in $\mathrm{dBm}$ ) as function of distance (in meters) for $900 \mathrm{MHz} 802.11$

\section{FACILITATING RAPID FIELD EXPERIMENTS}

Rapid progress in collection of measurement data depends on our capability of doing quick, inexpensive field experiments. There are multiple factors that can facilitate rapid field experiments, including flexible aircraft platform capabilities, staged and scripted experiments, flexible mobile lab to support flying and outdoor experiments, and separate teams for flying UAVs and for conducting communications and networking experiments. Below we discuss these factors.

\section{Flexible UAV Platforms}

several capable, Almost Ready to Fly (ARF) model aircraft platforms exist which are quite capable of carrying adequate test payloads. Advances in battery and motor technology have made electric flight a clean, easy, quiet option for test flying communications and networking payloads. Several platforms have been evaluated and tested for support of rapid, flexible experimentation. Two are outlined here. The Senior Telemaster, as depicted in Figure 3, is a 96" wingspan covered balsa aircraft with a lifting stabilizer. The aircraft provides adequate fuselage space for payloads, with a structure that offer many options for payload configuration. The SIG Rascal is 110" covered balsa aircraft with a large fuselage, easily handling a variety of payloads. Both of these platforms possess stable flight characteristics, with the ability to carry several pounds of payload. Power options include gas power, glow fueled engines, or electric motors. 
While small platform airborne testing can be done using conventional RC model aircraft control, the use of an autopilot greatly enhances the ability to perform effective testing. It eases the workload of test personnel, while generally providing the ability for better monitoring of aircraft parameters, effective automation of test flights, as well as better repeatability of test conditions. Some of our testing has made use of Procerus' Kestrel autopilot. The unit's ease of installation, configuration and use provides a capable system, effectively support a variety of tests, and eases pilot workload during the performance of tests.

\section{Staged and Scripted Experiments}

Even the simplest of experiments is complicated when we move from a laboratory environment to the field. As indicated earlier in our discussion on UAV networking experiments, a set of staged experiments that gradually build up the capabilities of performing experiments are useful. In order to maintain organization, and support smooth performance of test scenarios, it would be useful to make use of checklists and scripted procedures for execution of tests.

\section{Flexible Ground Support Infrastructure}

The availability of COTS aircraft platforms and wireless equipment provides relatively simple tools with which to test and analyze communications technologies. However, as with any work based on field experimentation, the amount of equipment, software and ancillary items required rapidly escalates. Note also that fly related operations are typically performed in more remote areas. A method for organization and transportation of assets is thus a key enabler of successful testing.

Among several solution options in this area, we will cover our use of a specially outfitted trailer, depicted in Figure 6, for field test support. The trailer contains support infrastructure (including battery recharging facilities), storage and workspace for the performance of a variety of communications and networking experiments.

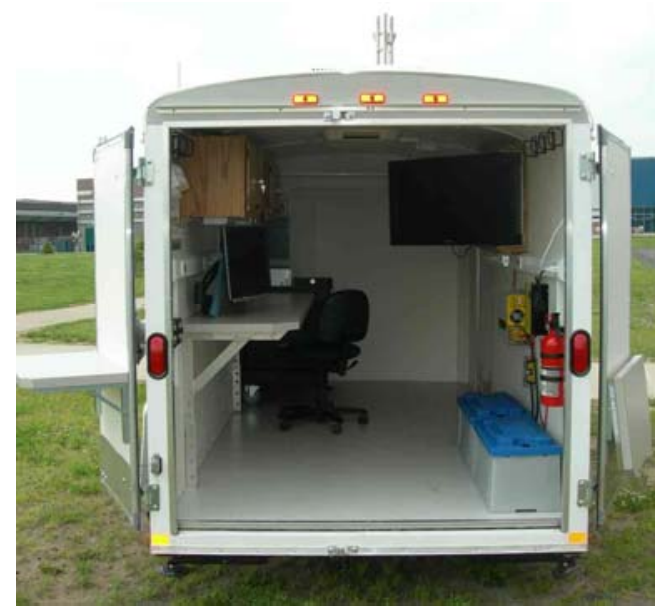

Figure 6. A mobile test support trailer to facilitate field experiments in UAV networking

The basic trailer is a standard commercial $6 \times 12$ foot enclosed single axle trailer. Support infrastructure consists climate control, as well as power distribution. "Shore Power" connections connect to commercial AC or generators. The trailer also contains a DC power system, drawing power from deep cycle batteries to provide AC power. This capability provides electrical support for short duration testing without requiring generator operation.

Processing, networking and communications resources include PC platforms, network hubs and wireless routers, and RF equipment. These resources can be rapidly reconfigured to support various testing requirements via patch panels and interconnects. This flexibility is fundamental to the trailers ability to support testing. As the types of communications being evaluated is constantly changing, communications capabilities, including RF units and antennas are swapped in and out as needed.

\section{A Separate Crew Responsible for Flying}

In our testing performed to this point, successful testing is a direct result of adequate manpower. More specifically, when utilizing UAVs, a separate crew for operation of the airborne platform as well as its maintenance and repairing improves the execution and safety of the testing. Isolating operation of the networking and communications payload from the operation of the platform allows personnel to focus on a specific aspect of the testing, providing improved oversight and test management. 


\section{FUTURE EXPERIMENTATION}

Many opportunities exist for future testing expanding on these experiments presented herein. In the area of communication link characterization, additional modems and radio solutions have been identified for comparison. Multiple antenna types will be characterized to determine their performance in typical small UAV environments. This information will be used to identify appropriate solutions for data connectivity to small UAV platforms, taking into account their characteristics and operating environments. Areas of specific interest include antenna placement, appropriate power levels, cost vs. range and dynamic antenna selection.

Testing and analysis of current wireless channel access protocols will proceed in a similar fashion to link analysis and testing. Additional work will be done in the characterization of the small UAV multi-hop environment. Future work will assess the characteristics of multi-hop architectures, measuring latencies, probability and duration for link connectivity, and end to end path characteristic in representative environments. Of special interest is the area of mobile ad-hoc and mesh network technologies, as well as emerging delay tolerant networking techniques.

\section{CONCLUSION}

Due to the availability of low-cost and yet highly capable COTS-based communications equipment and UAV platforms, it is now feasible to conduct rapid and inexpensive field experiments for UAV-based networks. These experiments can easily, and at low cost, yield test data that is more accurate and realistic than current simulation, modeling and assumptions. This accurate data is crucial to the development of novel UAV applications and networking protocols. In this paper we have described issues in designing UAV networking experiments, some ongoing field experiments, and methods of streamlining field experiments. We expect that these new experimental capabilities will significantly improve our knowledge on how to make best use of the UAV networking in the next several years.

\section{ACKNOWLEDGMENTS}

We would like to express our appreciation to those colleagues at AFRL and graduate students at Harvard who have helped design and implement the systems mentioned in this paper (see [4], [5] and [6]).

\section{REFERENCES}

[1] Basu, P., Redi, J., and V. Shurbanov, Coordinated Flocking of UAVs for Improved Connectivity of Mobile Ground Nodes, Proceedings of IEEE MILCOM 2004, Monterey, CA, November 2004.

[2] Brown, T. X., Argrow, B., Dixon, C., Doshi, S., Thekkekunnel, R-G., and D. Henkel, Ad hoc UAV-Ground Network (AUGNet), AIAA 3rd "Unmanned Unlimited" Technical Conference, Chicago, IL, 2004, pages 2023.

[3] Cerf, V., Burleigh, S., Hooke, A., Torgerson, L., Durst, R., Scott, K., Fall, K., and H. Weiss, DelayTolerant Network Architecture, Internet Draft, March 2006.

[4] Cheng, C-M, Hsiao, P-H, Kung, H. T., and D. Vlah, Adjacent Channel Interference in Dual-radio 802.11 Nodes and Its Impact on Multi-hop Networking, Proceedings of IEEE GLOBECOM 2006, San Francisco, California, November 2006.

[5] Cheng, C-M, Hsiao, P-H, Kung, H. T., and D. Vlah, Parallel Use of Multiple Channels in Multi-hop 802.11 Wireless Networks, Proceedings of IEEE MILCOM 2006, Washington, DC, October 2006.

[6] Cheng, C-M, Hsiao, P-H, Kung, H. T., and D. Vlah, Performance Measurement of 802.11a Wireless Links from UAV to Ground Nodes with Various Antenna Orientations, Proceedings of the 15th International Conference on Computer Communications and Networks (ICCCN 2006), Arlington, Virginia, October 2006.

[7] Das, S. R., Perkins, C. E., Royer, E. M., and M. K. Marina, Performance comparison of two on-demand routing protocols for ad hoc networks, Proceedings of IEEE INFOCOM, March 2000.

[8] Geer, H., and C. Bolkcom, Unmanned Aerial Vehicles: Background and Issues for Congress, CRS Report for Congress, Updated November 21, 2005.

[9] Li, J., Blake, C., De Couto, D, Lee, H. I., and R. Morris, Capacity of Ad Hoc Wireless Networks, Proceedings of the 7th ACM International Conference on Mobile Computing and Networking (MobiCom '01), Rome, Italy, July 2001, pages 61-69.

[10] Sklar, B., Digital Communications, Prentice Hall, 1988, p. 19 